\title{
Recognition and Management of Common Acute Conditions of the Oral Cavity Resulting From Tooth Decay, Periodontal Disease, and Trauma: An Update for the Family Physician
}

\author{
Paul C. Edwards, MSc, DDS, and Preetha Kanjirath, BDS, MDS
}

This article presents an overview of common and/or significant diseases of the oral cavity that the family physician is likely to encounter, with an emphasis on pathogenesis, recognition, complications, and management. Topics reviewed include the sequelae of dental caries, periodontal disease, and trauma. Prevention and early intervention strategies are emphasized. Recent updates and practical issues for the family physician are highlighted. (J Am Board Fam Med 2010;23:285-294.)

Keywords: Dental Decay, Otolaryngology, Periodontal Diseases

The oral cavity is a distinctive region of the body that, as a result of being exposed to the harsh oral environment, is subject to a distinctive set of conditions and diseases, many of which are unique to this area. Furthermore, the effect of disease in the oral cavity can have significant impact on the overall health of the individual.

\section{Dental Caries and Tooth Pain}

The teeth are 32 highly specialized hard tissue units that each consist of 3 functional units. First, the visible crown portion, which is exposed to the oral environment and is composed of an outer layer of inorganic, heavily mineralized tissue (enamel) overlying a somewhat softer collagen-containing calcified substance (dentin) that resembles bone in its ratio of organic to inorganic composition. Secondly, the root portion, which anchors the tooth to the surrounding alveolar bone by means of a dense,

This article was externally peer reviewed.

Submitted 3 February 2009; revised 30 November 2009; accepted 4 December 2009.

From the Department of Periodontics and Oral Medicine, Faculty of Dentistry, University of Michigan, Ann Arbor.

Funding: none.

Conflict of interest: none declared.

Corresponding author: Paul C. Edwards, MSc, DDS, Department of Periodontics and Oral Medicine, Faculty of Dentistry, University of Michigan, 1011 N University Avenue, Ann Arbor, MI 48109-1078 (E-mail: paulce@ umich.edu.) fibrous connective tissue attachment (periodontal ligament). Finally, a central pulp complex that is composed of neurovascular and loose, fibrous connective tissue.

Dental caries, or tooth decay, is one of the most prevalent chronic diseases affecting modern society. These are also known colloquially as cavities, reflecting the cavitation that occurs in more advanced lesions. Once viewed as a disease of children (Figure 1), it seems that, because a greater percentage of the adult population are retaining their teeth, adults are now developing new carious lesions at the same incidence rate as children. ${ }^{1}$ Among some methamphetamine abusers, a pattern of rampant carious destruction is noted, a condition termed "meth mouth" (Figure 2). There is also an exponential increase in risk of developing caries among patients who take multiple medications that predispose to xerostomia. Among the aging population, caries are commonly seen on the root surface of the teeth, where the gums have receded (Figure 3).

Caries develop through a complex interaction in which transmissible cariogenic oral microflora, primarily Streptococcus mutans and lactobacilli, metabolize fermentable dietary carbohydrates, resulting in lactic acid production. ${ }^{3}$ The resultant drop in $\mathrm{pH}$ at the tooth surface results in dissolution of the mineral component of the enamel. This process is 
Figure 1. Rampant caries in a child. (Photograph courtesy of Dr. Ana Veira.)

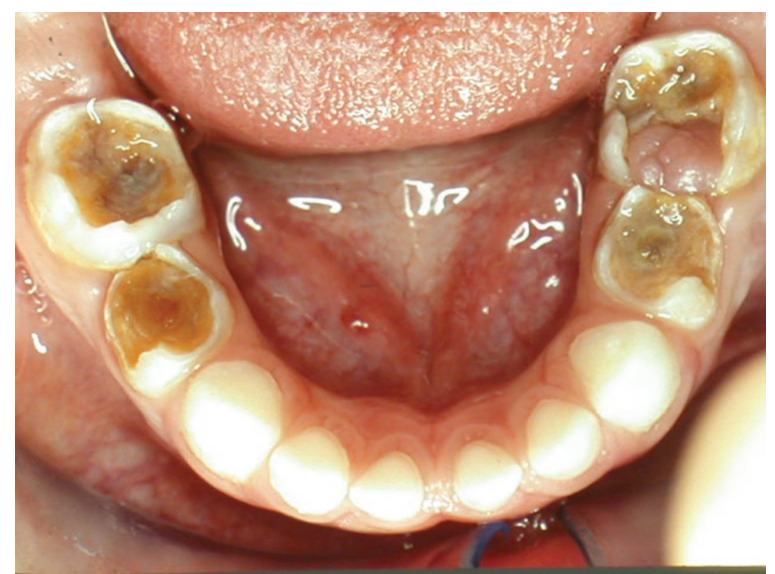

a dynamic one, in which demineralization is countered by remineralization through the buffering capacity of the saliva. ${ }^{4}$ Progression of caries results when the rate of demineralization exceeds the remineralization rate. Clinically, caries start in the deep pits on the biting surfaces of the posterior teeth or on the smooth surfaces of the teeth (Figure 4). Although often visible to the naked eye as a soft area of surface cavitation, ranging from white (reflecting early demineralization) to brown or black in color, the caries process can also occur beneath an intact layer of surface enamel or between 2 adjacent teeth, making early visual recognition extremely difficult without the assistance of radiographs (Figure 5).

Although the caries process in the heavily mineralized enamel layer of the tooth is primarily a physicochemical process, the underlying dentin is connected to the highly innervated pulp tissue at

Figure 2. Rampant caries in a methamphetamine user.

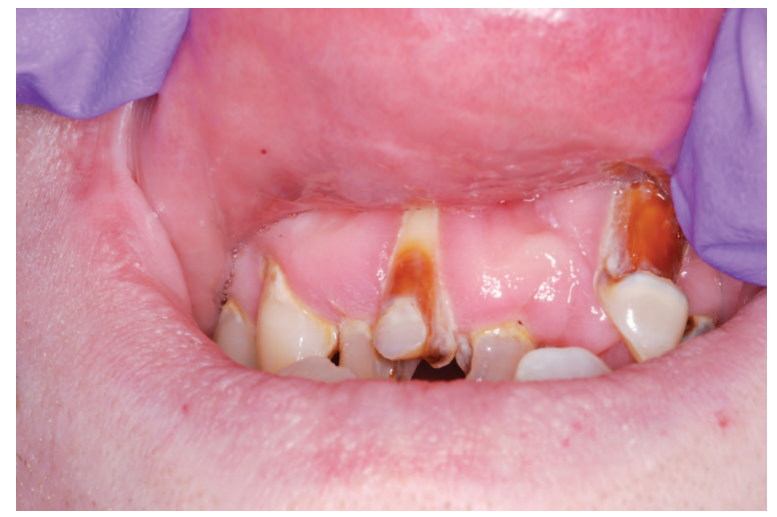

Figure 3. Root caries may be difficult to detect clinically because they are frequently hidden by the gingiva. (Photograph courtesy of Dr. Eduardo Bresciani.)

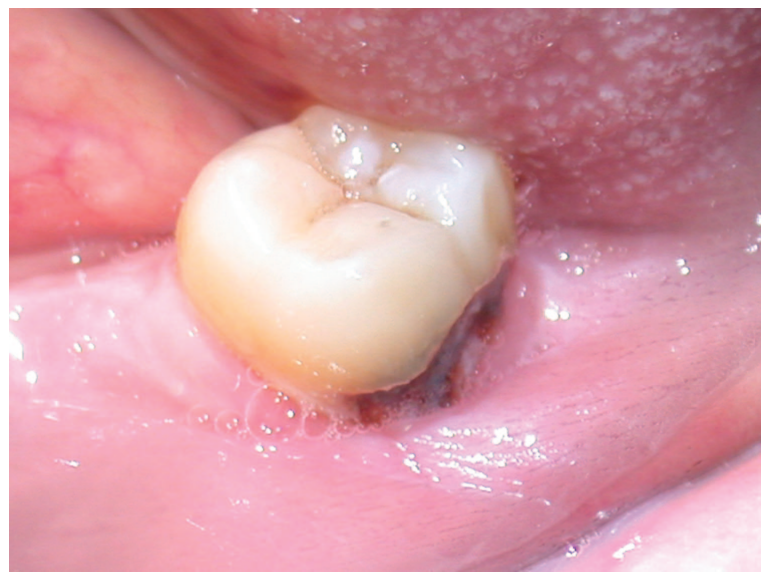

the center of the tooth through small-diameter dentinal tubules. When caries penetrate through the full thickness of the enamel to reach the underlying dentin, patients will typically exhibit momentary tooth pain on exposure to cold and/or osmotic agents such as sugar. This process, termed reversible pulpitis, is not an acute emergency and can typically be treated by conservative mechanical removal of the decay and placement of a dental restoration (a "filling") to replace the lost tooth structure, provided that this treatment is initiated in a reasonable time frame (typically several weeks). A similar type of pain can be elicited in patients who have gingival

Figure 4. Caries commonly start in the deep grooves (pits and fissures) located on the biting surface of the molar teeth. In this case, a small surface opening hides extensive subsurface decay. (Photograph courtesy of Dr. Eduardo Bresciani.)

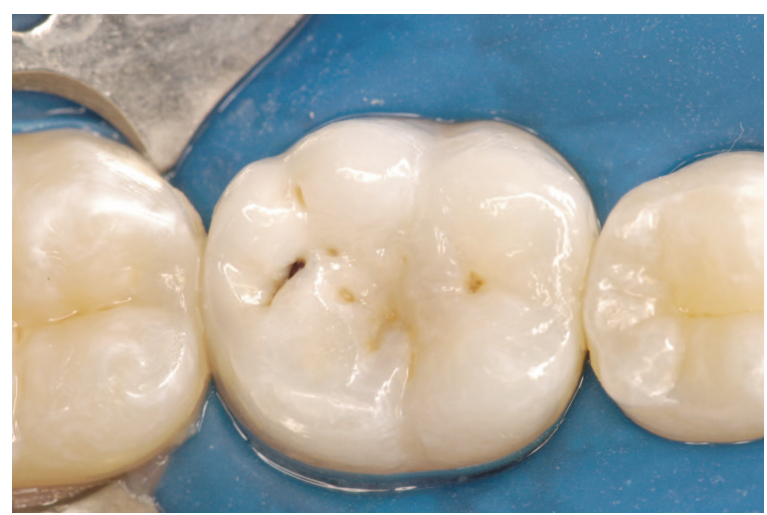


Figure 5. This radiograph shows interproximal carious lesions that would have been difficult to detect by clinical examination alone.

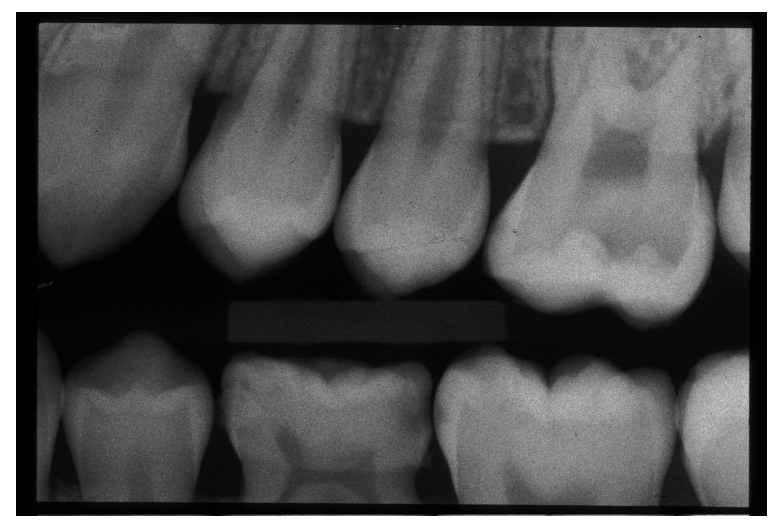

recession, resulting from either excessively rough tooth brushing habits or as a result of periodontal disease. The exposed root surface, especially when coupled with toothbrush-related removal of the protective cementum, results in momentary tooth pain on exposure to cold and/or osmotic agents such as sugar. The degree of sensitivity can be reduced by instructing the patient to change their brushing technique and use less force while brushing, incorporating a vertical brushing stroke instead of a "back and forth" horizontal brushing motion, coupled with long-term use of specially formulated toothpaste for "sensitive" teeth. These toothpastes contain salts such as potassium nitrate or potassium oxalate that seal the exposed dentinal tubules and/or modulate nerve transmission. Dentist-applied agents such as fluoride varnishes and thin layers of bonded resin can provide more rapid resolution of symptoms.

If caries go untreated, severe dental pain that either persists after removal of the inciting stimulus or occurs spontaneously will typically result. This condition, termed irreversible pulpitis, results in severe pain that may last for hours or days if untreated. Patients with irreversible pulpitis will typically present on an urgent basis in acute distress with minimal physical reserves after being unable to obtain any relief for an extended period of time. The pain is typically generalized, involving the area of several teeth, and may even be referred to the opposing jaw, often making precise identification of the offending tooth by the patient or provider difficult. Patients should be offered palliative treatment for pain. Although nonsteroidal anti-inflam- matory agents (eg, ibuprofen 600 to $800 \mathrm{mg}$ every 4 to 6 hours) may be beneficial in cases of mild to moderate pain, in more severe cases acetaminophen-narcotic combinations (eg, Tylenol \#3, Percocet, or Vicodin) are typically required. Although antibiotics are often inappropriately prescribed, they are not indicated at this stage. Instead, it is important to refer the patient to a dental provider. Definitive treatment involves either surgical extraction of the involved tooth or, if the decision is made to try to preserve the tooth, mechanical extirpation of the pulpal tissue (a pulpectomy; the first step in root canal treatment).

Eventually, if untreated, pulpal necrosis will develop as a result of prolonged inflammation within the confined space of the pulp cavity. This condition, termed acute apical periodontitis, is characterized by severe pain located to the affected tooth. The pain is aggravated by pressure (eg, touch or biting). As with irreversible pulpitis, treatment involves prompt root canal therapy or extraction of the tooth. Because the pain is the result of necrotic pulp tissue and not an infectious process, antibiotic therapy is not indicated. ${ }^{5,6}$ However, in the presence of underlying medical conditions that compromise host resistance, such as poorly controlled diabetes or among patients taking high-dose corticosteroids, antibiotic therapy, as described below for an acute apical abscess, should be considered.

In many cases, depending on the host's immune response, bacterial load, and virulence, inflammation may spread beyond the apical area of the tooth, resulting in an acute apical abscess. This is evident clinically by the presence of an intraoral swelling, typically located within the buccal vestibule apical to the tooth root. This situation is ideally managed by a dentist through removal of the tooth or extirpation of the necrotic pulp tissue and/or incision and drainage of any fluctuant abscess. If immediate dental management cannot be arranged, empirical broad-spectrum antibiotic coverage should be considered, especially if the patient has any underlying medical conditions that could reduce bacterial resistance (eg, diabetes, inability to sleep because of severe dental pain, alcoholism, etc).

Dental infections are typically caused by normal oral flora. During the first 3 days of the process, facultative Gram-positive oral streptococci predominate. The antibiotic of choice during this early period is penicillin VK $500 \mathrm{mg}$ by mouth every 6 hours for 7 days. $^{7,8}$ Amoxicillin-500 mg every 8 
hours-is an acceptable alternative. If the swelling has been present for more than 3 days or is unresponsive to penicillin therapy, metronidazole ( 250 to $500 \mathrm{mg}$ by mouth every 6 hours) may be added. ${ }^{9}$ For a patient who is allergic to penicillin, clindamycin $300 \mathrm{mg}$ by mouth every 6 hours for 7 days is recommended. 9

This infection has the potential to develop into a true medical emergency if not treated promptly and aggressively. Cellulitis, characterized by painful swelling, regional lymphadenopathy, and in many cases fever, may rapidly develop if the infection is allowed to spread into the surrounding fascial planes. Serious complications can include compromised breathing (Ludwig angina) for infections of mandibular origin and orbital or cavernous sinus involvement for maxillary infections. Although rare, death can occur, especially among healthy young adults. ${ }^{10}$ Any sign of fascial plane involvement necessitates immediate referral to an oral and maxillofacial surgeon or a hospital dental department. If any signs of respiratory difficulty are apparent, the patient should be referred directly to the nearest emergency department.

Fortunately, in most cases, the necrotic tooth follows a less fulminant clinical course, progressing instead to a chronic low-grade inflammatory process involving the apical supporting bone. Degradation products from bacteria and necrotic pulp tissue lead to chronic bone destruction and the formation of a mass of chronically inflamed granulation tissue within the jaw, termed chronic apical periodontitis. In long-standing lesions, mediators of inflammation may trigger a proliferation of dormant epithelial cells in the area of the root apex, resulting in the formation of a periapical cyst. Clinically, the involved teeth are usually asymptomatic, although a slight increase in mobility and mild discomfort may be elicited by tapping on the biting surface of the tooth. An area of bone resorption is typically noted around the apex of the tooth root following radiographic examination.

Definitive treatment involves extraction of the causative tooth with conservative curettage of any remaining lining from the cyst cavity. When sufficient tooth structure remains to allow for restoration of the tooth, endodontic treatment can be attempted. ${ }^{11}$ Failure to treat these lesions will lead to continued bone destruction and eventual perforation of the cortical plates of bone. In exceedingly rare cases, the epithelial lining can undergo malig-
Figure 6. Pictorial representation of the components of a normal periodontium with healthy tissues. (Diagram courtesy of Chris Jung.)

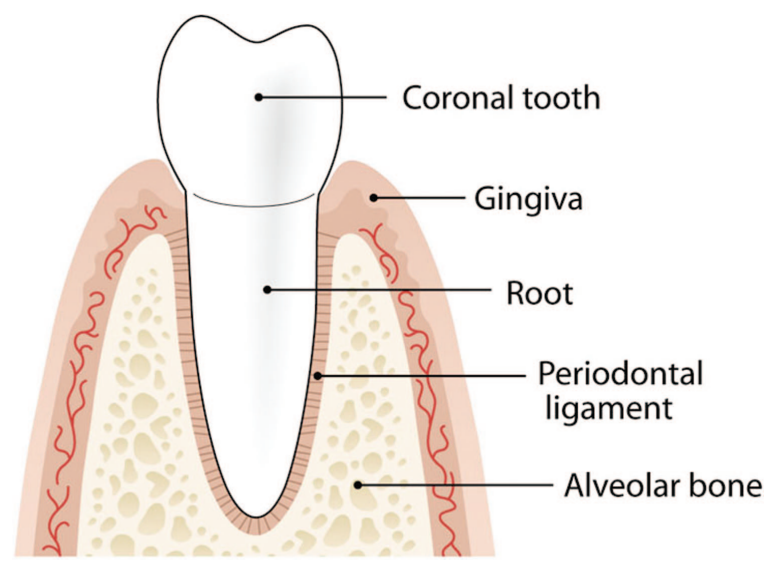

nant transformation. ${ }^{12}$ More commonly, this chronic process will continue with only sporadic mild symptoms until it is recognized by the patient's dentist. In many cases, the patient will eventually develop an acute exacerbation of chronic apical periodontitis, which clinically resembles an acute apical abscess. Typically, the patient will report waking up in the morning with a prominent swelling and in severe pain resulting from tissue expansion. Treatment involves empirical antibiotic therapy as previously described until the patient can be definitively treated.

\section{Diseases of the Structures That Support the Teeth}

The periodontal complex (Figure 6) consists of the gingiva; cementum (an acellular, bone-like hard tissue that covers the tooth root); the periodontal ligament (a vascular connective tissue that envelops the tooth and connects it to the jaws, ${ }^{13}$ forming a hammock-like structure); and the alveolar bone. In the periodontally healthy adult, the masticatory mucosa covers the alveolar bone and the tooth root just coronal to the cementoenamel junction.

Periodontitis refers to a complex process involving the periodontal supporting structures and results from breakdown of the connective tissue and the supporting alveolar bone in response to inflammation after bacterial colonization. Gram-negative bacteria such as actinobacillus actinomycetemcomitans, porphyromonas gingivalis, and spirochetes ${ }^{14}$ are the most common microbial agents implicated in periodontitis. Within the adherent plaque, these bac- 
Figure 7. Schematic representation of periodontal disease showing tissue destruction and alveolar bone loss. (Diagram courtesy of Chris Jung.)

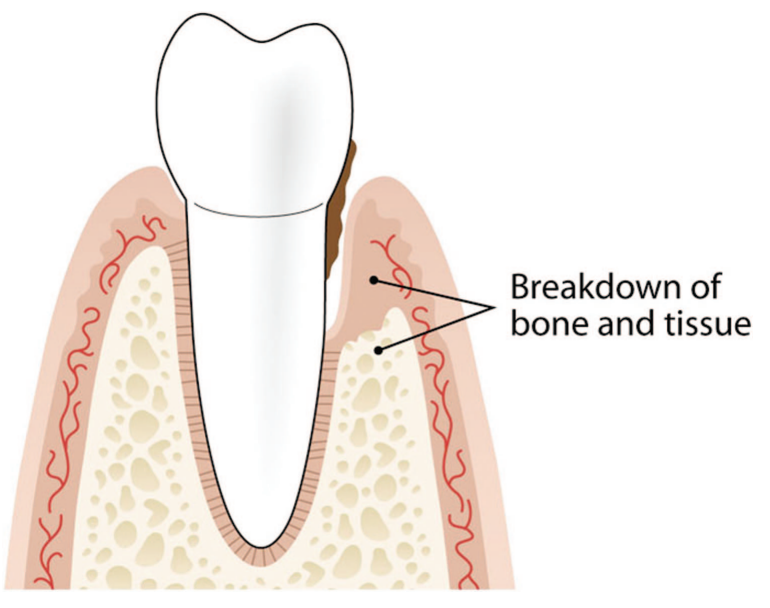

teria stimulate the production of inflammatory cell mediators (including interleukins, tumor necrosis factor $\alpha$, prostaglandin $\mathrm{E}_{2}$ and matrix metalloproteinases), which in turn reduce the production of new collagen fibers resulting in tissue destruction. ${ }^{15}$ Although the host inflammatory response to superficial bacterial colonization ("plaque") is an essential component to the development of periodontal disease, this response is modified by both local and systemic factors. Local etiologic factors include calculus (calcified plaque deposits along the roots of teeth), deep gingival pockets around the teeth that make removal of bacterial deposits by the patient through routine home care more difficult, less than ideal spacing between teeth, xerostomia, and trauma from occlusion. Systemic factors that may predispose an individual to periodontal breakdown include congenital conditions such as Down syndrome and Papillon-Lefevre syndrome ${ }^{16,17}$ and acquired conditions such as diabetes. ${ }^{14}$ Although definitive evidence is lacking at this point, there is a growing body of research that suggests that the presence of chronic inflammation from periodontal disease may exacerbate the progression of systemic conditions such as diabetes ${ }^{18}$ and cardiovascular disease ${ }^{19,20}$ and may be associated with an increased risk of preterm labor. ${ }^{21,22}$

From the aspect of patient management, earlystage periodontitis is a chronic condition that usually presents with few overt visual signs of disease activity other than increased bleeding when brushing. It often involves only a few teeth at a time. By the time the degree of attachment loss has pro- gressed to the point of tooth mobility, the prospects of a favorable outcome (ie, long-term tooth preservation) are guarded.

As periodontal disease progresses, the loss of supporting tissues between the tooth root and alveolar bone leads to the creation of defects known as periodontal pockets (Figure 7). Proliferation of mixed anaerobic bacteria within a periodontal pocket may lead to a localized purulent inflammation termed a periodontal abscess, which is characterized by suppuration from the periodontal pocket accompanied by pain, tenderness, swelling, and tooth mobility (Figure 8). Periodontal abscesses are typically noted in otherwise healthy patients with untreated periodontal disease. ${ }^{23}$ Patients with a reduced ability to combat infection as a result of underlying systemic diseases such as diabetes are at a higher risk of developing periodontal abscesses. Periodontal abscesses can also result from the introduction of a foreign body into the periodontal pocket (eg, a popcorn husk) or from mechanical injury to the periodontal lining after routine dental scaling.

Clinically, the differential diagnosis of a localized abscess involving a single tooth also includes the acute apical abscess from a necrotic tooth (see above for details). Periodontal abscesses typically are characterized by mild to moderate pain, whereas abscesses of pulpal origin are associated with a greater degree of pain. Reaching a definitive diagnosis is not easily accomplished in a family physician's office. A definitive diagnosis is accomplished by demonstrating suppuration from the periodontal pocket in the presence of a vital tooth. Initial management of a periodontal abscess will

Figure 8. Periodontal abscesses involving 2 neighboring teeth, resulting in draining fistulous tracts.

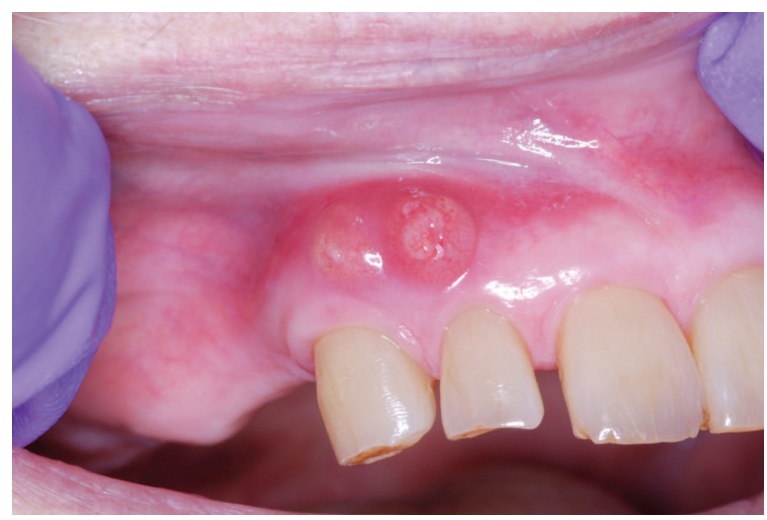


depend on the severity of the infection and involves both pain management and local intervention ranging from incision and drainage to extraction of the tooth. Systemic antibiotics are reserved for rare cases involving cellulitis and systemic symptoms. Definitive management requires referral to a dentist for treatment of the underlying periodontal disease, which typically involves the mechanical removal of calcified deposits (calculus) through processes known as scaling and root planing, and, in certain cases, systemic or locally applied antibiotics.

Necrotizing ulcerative gingivitis, also known as "trench mouth," is an acute inflammatory condition of the gums caused by anaerobic bacteria (primarily treponema, selenomonas, fusobacterium, and prevotella intermedia). This condition typically presents in young adults under stress in whom host defenses are compromised by poor nutrition and poor oral hygiene. Clinically, the gingival tissue is denuded with punched out, crater-like areas of necrosis and blunting of the interdental papilla. This condition is accompanied by pain, fetid odor, and systemic symptoms of fever, malaise, and cervical lymphadenopathy.

Patients should be prescribed penicillin VK 500 mg orally every 6 hours for 7 days. ${ }^{24}$ In patients with a penicillin allergy, erythromycin $500 \mathrm{mg}$ or metronidazole $500 \mathrm{mg}$ twice daily for 7 days is recommended. Home care involves gentle cleansing of the involved areas using frequent rinses of chlorhexidine gluconate and salt water. Referral to a dental practitioner for debridement and definitive periodontal therapy is recommended. An exceedingly rare but severe presentation of this condition, termed noma (from the Greek numein, meaning "to devour"), in which the area of necrosis extends past the gingival margins, may occur in patients with advanced acquired immune deficiency syndrome or who are otherwise severely immunocompromised or malnourished.

Pericoronitis is an acute gingival condition characterized by inflammation around the tissue of a partially erupting tooth. This typically occurs in young adults. The pathogenesis of this condition is analogous to the periodontal abscess. Entrapment of debris between the tissue overlying the partially erupted tooth (the operculum) and the tooth permits the growth and spread of anaerobic bacteria (Figure 9). ${ }^{25}$ The inflamed operculum is often secondarily traumatized by the opposing tooth, further aggravating the situation.
Figure 9. Early pericoronitis associated with partially erupted third molar. Note the soft tissue coverage over the distal portion of the third molar (operculum). In this case, the gingiva was slightly erythematous. No associated swelling was noted, however, purulent exudate was expressible from the pocket. Also note the caries on the partially erupted third molar. (Photograph courtesy of Dr. Pilar Hita-Iglesias.)

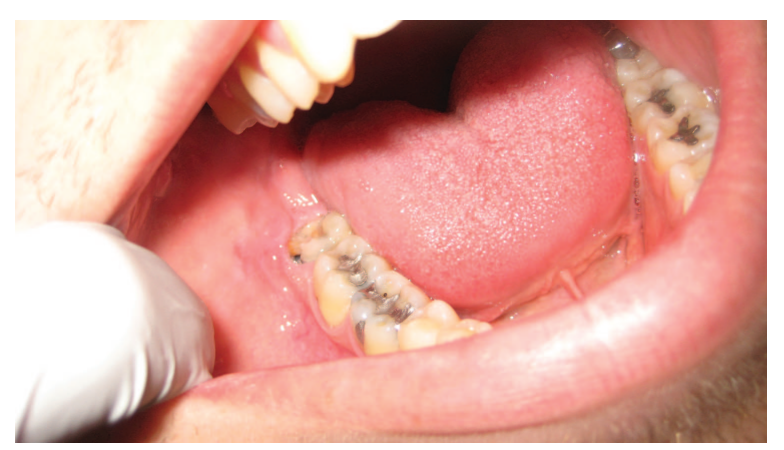

Pericoronitis is accompanied by pain radiating to the ipsilateral ear and throat. Severe cases may be accompanied by fever, malaise, lymphadenopathy, and trismus. Clinically, a partially erupted third molar covered on the distal aspect by erythema and inflamed gingiva with associated swelling and/or purulence is noted. If untreated, infection can progress to the submandibular and sublingual spaces. If any evidence of airway compromise is noted, immediate referral for emergency airway management and drainage are indicated. Fortunately, this outcome is rare.

Management options include surgical removal of the operculum or irrigation with $2 \%$ chlorhexidine gluconate and debridement of the tissues. The patient can also be instructed to irrigate under the operculum with warm salt water or $0.12 \%$ chlorhexidine. Antibiotic therapy with penicillin VK is recommended. Definitive treatment involves removal of the partially erupted third molars.

\section{Nonodontogenic Mimickers of Dental Pain}

The vast majority of symptoms experienced within the oral cavity are related to the conditions discussed above (Figure 10). However, the clinician must always consider the possibility of pain of nondental origin when assessing the patient's chief complaint. The most common cause of referred pain to the posterior maxillary teeth is maxillary sinusitis. Typically, the pain is of mild to moderate intensity, and usually involves 2 to 3 posterior max- 
Figure 10. Differential diagnosis of common causes of dental pain. Additional clinical information and treatment recommendations are provided in the text.

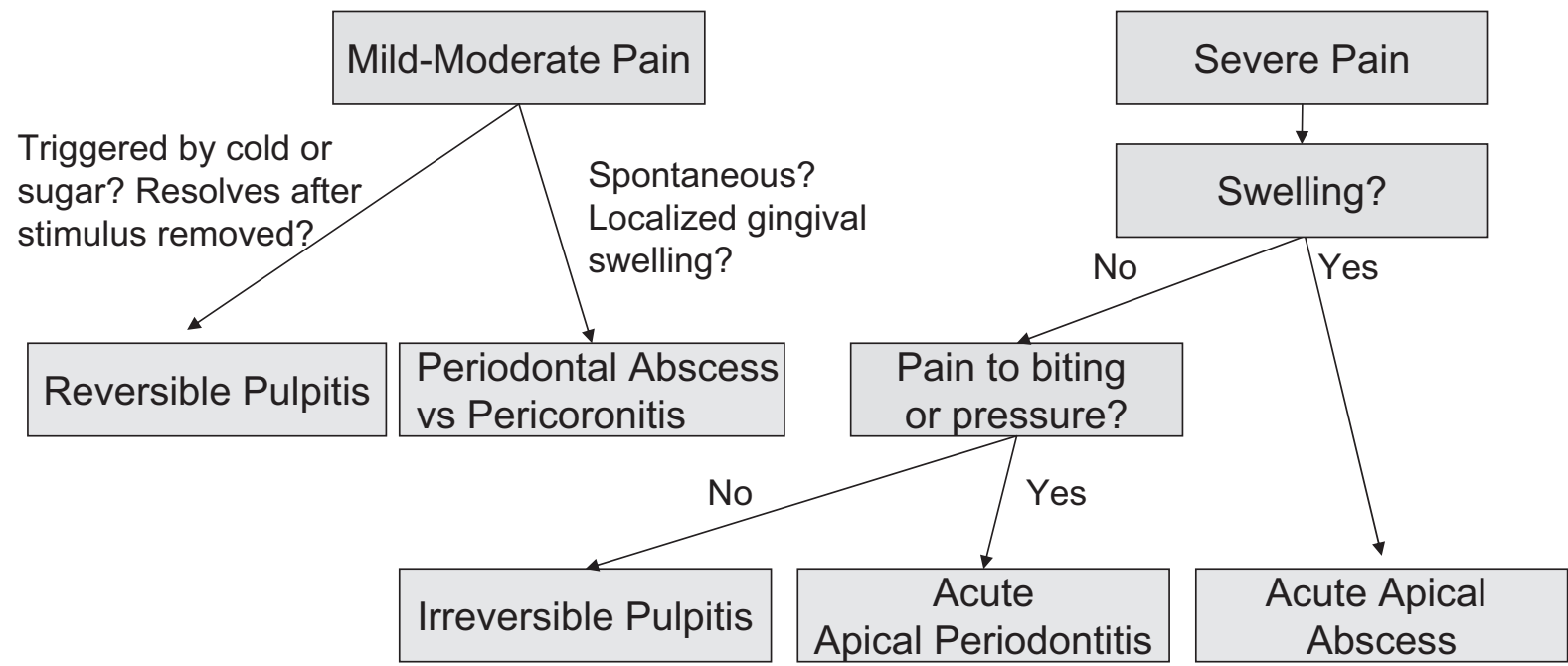

illary molar teeth. The patient will report increased pain when bending over or if asked to firmly press down on their feet. Other common conditions that may present as oral pain that can be mistaken for pain of dental origin include temporomandibular joint disorders and aphthous ulcers. Rare mimickers of dental pain include referred myocardial pain, atypical facial pain syndromes, and maxillary sinus malignancies.

\section{Dental Trauma}

Dental trauma is extremely common, with an estimated $10 \%$ to $16 \%$ of children exhibiting evidence of damage to the permanent incisor teeth by age $15 .{ }^{26}$ Dental trauma is particularly common in the 6- to 12-year-old age group. Depending on the angle and the magnitude of force, trauma may result in injuries ranging from fracture of the crown to concussive, intrusive-, or extrusive-type injuries to the tooth to outright fractures of the alveolar process or the jaws. Fortunately, the root is supported by a fibrous periodontal ligament, rendering it able to absorb mild to moderate forces. This may in part account for the observation that the most common permanent injury among teeth subjected to trauma is the fracture of an incisal portion of the crown. ${ }^{26}$

Although all types of injury to the maxillofacial complex require prompt evaluation by a dentist, it is essential that all patients with avulsive injuries of permanent teeth be treated immediately. Studies have shown that if the tooth is reimplanted within
60 minutes, the success rate of reimplantation is dramatically improved. ${ }^{27}$ The ideal situation is if the patient or a caregiver can reimplant the tooth at the time of injury, providing the tooth or socket have not been visibly soiled. If not, the goal of managing such a scenario is to keep the cells of the periodontal ligament viable by keeping them moist until the tooth can be reimplanted. The ideal storage/transport medium is Hanks balanced salt solution, which is available commercially in a kit form as "Save-A-Tooth" (Phoenix-Lazerus, Inc., Pottstown, PA). Other good options include placing the tooth in milk or having the patient keep the tooth in the mouth, positioned against the cheek. Storage in plain water or saline is to be avoided, although it is still preferable to desiccation. When the patient arrives at the practitioner's office, the tooth should be reimplanted immediately with minimal manipulation of the root tissues. If necessary (for example, if the tooth root was visibly soiled), the tooth and socket can be rinsed carefully with a cell-preserving media. Once the tooth has been reinserted, the clinician should carefully compress the alveolar bone around the socket with moderate finger pressure and arrange for immediate referral to a dentist. Depending on how long the tooth has been out of the socket and the degree of closure of the root apex, the dentist will decide on the need for additional tooth preparation before reimplantation. The dentist will then stabilize the tooth to the neighboring teeth using a bonded splint. The patient should be evaluated for concussive injuries to 
the brain and fractures of the facial bones and/or mandible. The patient should also be assessed for the need for tetanus prophylaxis, especially if the vaccination status of the patient is unclear or if the tooth has come into contact with soil. ${ }^{28}$ The American Academy of Endodontics ${ }^{28}$ recommends that patients with a reimplanted tooth be placed on doxycycline (adult dosage of $100 \mathrm{mg}$ twice a day) for 7 days. As an alternative, if staining of developing teeth is a concern, penicillin VK (adult dosage: 1 to $2 \mathrm{~g}$ immediately, then $500 \mathrm{mg}$ four times per day) is an option. Based on the "dry time" and the degree of closure of the root apex, the patient's dentist will decide whether endodontic therapy is also required. Primary teeth should not be reimplanted because of the risk of damage to the underlying developing permanent tooth. If in doubt as to whether a tooth is a primary tooth (some primary teeth are typically present up to 12 to 14 years of age), the physician should reimplant it if possible and arrange for immediate dental assessment.

\section{Prevention of Oral Diseases}

As with most conditions, prevention and early recognition/treatment offer the greatest potential to reduce the incidence and severity of dental disease. From the family physician's vantage point, a number of observations are offered:

- Reduced salivary flow, a common side effect of many drugs, is associated with a loss of buffering activity and hence an increased risk of caries and mucosal infections. Common xerostomia-inducing drugs include anticholinergics, antidepressants, antipsychotics, diuretics, antihypertensives, antihistamines, narcotics, and anxiolytics. ${ }^{29}$ This is a significant problem among the older population, especially in view of the trend toward more patients keeping their teeth. This should be taken into account when prescribing for patients in this population, especially when contemplating polypharmacy. When the use of alternative drugs is not feasible, cholinergic agents such as pilocarpine (5 $\mathrm{mg}$ three times daily) or cevimeline (30 $\mathrm{mg}$ three times daily) may be considered. A number of commercially available saliva substitutes are available over the counter. Many patients with severe xerostomia experience relief from carrying a bottle of water with them to sip as needed. Home applica- tion of gels and/or rinses containing fluoride should be considered for patients in this group (please see number 6, below). As for all high-risk patients, increased dental vigilance is needed.

- Many orally administered medications contain high concentrations of sweetening agents to make them more palatable. In vulnerable pediatric populations with underlying chronic conditions, prolonged exposure to these agents can result in a significant increase in caries development. $^{30}$

- Because cariogenic oral microflora can be vertically transmitted to a child through the mother's saliva, management of active caries in women of childbearing age, pregnant women, mothers, and caretakers of young children should be encouraged. $^{31}$

- Routine dental examinations and radiographic evaluations of the teeth should be strongly encouraged because these are crucial to the early detection and treatment of carious lesions that may not be evident on a clinical examination.

- Management of chronic periodontal infection will reduce systemic spread of bacterial endotoxins and inflammatory mediators, thus potentially reducing their negative impact on chronic disease conditions.

- The risk of developing caries can be reduced by oral hygiene practices such as brushing and flossing, which reduce the tooth surface biofilm, and dietary counseling to reduce the frequency of sugar consumption. In patients unable to floss effectively, one short-term study demonstrated that daily oral irrigation with a dental water jet combined with brushing may be as effective as brushing and flossing at reducing gingival bleeding, inflammation, and plaque accumulation. ${ }^{32}$ Exposure of enamel to trace quantities of fluoride ion $(\sim 1 \mathrm{ppm})$ results in the formation of fluoridated hydroxyapatite crystals that demonstrate increased resistance to acid dissolution. The overall magnitude of caries reduction averages $25 \%$, whether delivered professionally, self-administered through toothpaste, or by community water fluoridation. ${ }^{1}$ Fluoride brush-on gels with a neutral pH (eg, Prevident 1.1\% brush-on gel, Colgate Professional) are preferred over acidulated gels for daily, at-home therapy. Weekly use of a $0.2 \%$ neutral sodium fluoride gel should also be considered for use by high-risk individuals. 
- Pit and fissure sealants (resinous material that is placed into the deep crevices on the biting surfaces of the molar teeth without the need for drilling, creating a barrier to the entry of bacteria) are effective at preventing caries on these tooth surfaces, especially in patients at a high risk for caries. This caries protection ranges from close to $100 \%$ protection in ideal situations, in which long-term retention of the sealant is maintained with periodic re-examination and reapplication where needed, ${ }^{33}$ to $75 \%$ in "real world" situations. ${ }^{34}$ Because close to $90 \%$ of all cavities involving the permanent teeth of school-age children occur on the occlusal surfaces of the molars, the application of sealants can play a significant role in reducing the caries rate, especially among children with a history of high caries rate in the primary dentition. Therefore, parents of children with newly erupting molar teeth (approximately 6 to 7 and 11 to 12 years of age) should be encouraged to visit their primary dental care provider for assessment of whether sealants are indicated.

- The lifetime direct financial cost for treatment of a single tooth lost to trauma as a child or teenager easily runs into the $\$ 10,000+$ range. The use of custom mouth guards should be encouraged for all patients performing any type of physical activity in which accidental trauma to the jaws is a possibility. This includes not just high-impact sports such as boxing, hockey, and football, but also physical sports such as soccer, baseball, and basketball that traditionally are viewed as being less aggressive. A recent meta-analysis concluded that wearing a mouthguard during sports-related activities reduced the risk of an orofacial injury by 1.6 to 1.9 times. ${ }^{35}$ However, the evidence as to whether mouthguards protect against concussive injuries was inconclusive. Although not as effective and not as comfortable to wear as custom mouth guards, over-the-counter products are an acceptable, lower-cost alternative.

\section{References}

1. Griffin SO, Regnier E, Griffin PM, Huntley V. Effectiveness of fluoride in preventing caries in adults. J Dent Res 2007;86:410-5.

2. Shaner JW, Kimmes N, Saini T, Edwards PC. Meth mouth: rampant caries in methamphetamine abusers. AIDS Patient Care STDS 2006;20:4-8.

3. Loesche WJ. Role of Streptococcus mutans in human dental decay. Microbiol Rev 1986;50:353-80.
4. Selwitz RH, Ismail AI, Pitts NB. Dental caries. Lancet 2007;369:51-9.

5. Nagle D, Reader A, Beck M, Weaver J. Effect of systemic penicillin on pain in untreated irreversible pulpitis. Oral Surg Oral Med Oral Pathol Oral Radiol Endod 2000;90:636-40.

6. Keenan JV, Farman AG, Fedorowicz Z, Newton JT. A Cochrane systematic review finds no evidence to support the use of antibiotics for pain relief in irreversible pulpitis. J Endod 2006;32:87-92.

7. Sweeney LC, Dave J, Chambers PA, Heritage J. Antibiotic resistance in general dental practice: a cause for concern? J Antimicrob Chemother 2004; 53:567-76.

8. Warnke PH, Becker ST, Springer IN, et al. Penicillin compared with other advanced broad spectrum antibiotics regarding antibacterial activity against oral pathogens isolated from odontogenic abscesses. J Craniomaxillofac Surg 2008;36:462-7.

9. Ellison SJ. The role of phenoxymethylpenicillin, amoxicillin, metronidazole and clindamycin in the management of acute dentoalveolar abscesses: a review. Br Dent J 2009;206:357-62.

10. Otto M. Boy's death fuels drives to fund dental aid to poor. Washington Post. March 3, 2007;B01.

11. Caliskan MK. Prognosis of large cyst-like periapical lesions following nonsurgical root canal treatment: a clinical review. Int Endod J 2004;37:408-16.

12. Whitlock RI, Jones JH. Squamous cell carcinoma of the jaw arising in a simple cyst. Oral Surg Oral Med Oral Pathol 1967;24:530-6.

13. McCulloch CA, Lekie P, Mc Kee MD. Role of physical forces in regulating the form and function of the periodontal ligament. Periodontol 2000 2000;24: $56-72$.

14. Consensus report. Periodontal diseases: pathogenesis and microbial factors. Ann Periodontol 1996;1: 926-32.

15. Page RC. The pathobiology of periodontal diseases may affect systemic diseases; inversion of a paradigm. Ann Periodontol 1998;3:108-20.

16. Kinane DF. Blood and lymphoreticular disorders. Periodontol 2000 1999;21:84-93.

17. Kinane DF. Periodontitis modified by systemic factors. Ann Periodontol 1999;4:54-64.

18. Taylor GW, Borgnakke WS. Periodontal disease association with diabetes, glycemic control and complications. Oral Dis 2008;14:204-5.

19. Beck J, Eke P, Heiss G, et al. Periodontal disease and coronary heart disease: a reappraisal of the exposure. Circulation 2005;112:19-24.

20. Beck J, Garcia R, Heiss G, Vokonas PS, Offenbacher S. Periodontal disease and cardiovascular disease. J Periodont 1996;67:1123-37.

21. Bobetsis YA, Barros SP, Offenbacher S. Exploring the relationship between periodontal disease and 
pregnancy complications. J Am Dent Assoc 2006; 137(Suppl):7S-13S.

22. Scannapieco FA, Bush RB, Paju S. Periodontal disease as a risk factor for adverse pregnancy outcomes. A systematic review. Ann Periodontol 2003;8:70-8.

23. Herrera D, Roldan S, Mariano S. The periodontal abscess; a review. J Clin Periodontol 2000;27:37786.

24. Wade AB, Blake GC, Mirza KB. Effectiveness of metronidazole in treating the acute phase of ulcerative gingivitis. Dent Pract 1966;16:440-4.

25. Maloney J, Stassen LF. The relationship between pericoronitis, wisdom teeth, putative periodontal pathogens and the host response. J Am Dent Assoc 2008;54:134-7.

26. Chadwick BL, White DA, Morris AJ, Pitts NB. Non-carious tooth conditions in children in the UK, 2003. Br Dent J 2006;200:379-84.

27. Blomlöf L, Andersson L, Lindskog S, Hedström KG, Hammarström L. Periodontal healing of replanted monkey teeth prevented from drying. Acta Odontol Scand 1983;41:117-23.

28. American Association of Endodontists. Recommended guidelines of the American Association of Endodontists for the treatment of traumatic dental injuries. Chicago, IL: American Association of Endodontists; 2003.
29. Moore PA, Guggenheimer J. Medication-induced hyposalivation: etiology, diagnosis, and treatment. Compend Contin Educ Dent 2008:29:50-5.

30. Bigeard L. The role of medication and sugars in pediatric dental populations. Dent Clin North Am 2000:44;443-56.

31. Napimoga MH, Hofling JF, Klein MI, Kamiya RU, Goncalves RB. Transmission, diversity and virulence factors of Streptococcus mutans genotypes. J Oral Sci 2005;47:59-64.

32. Barnes CM, Russell CM, Reinhart RA, Payne JB, Lyle DM. Comparison of irrigation to floss as an adjunct to toothbrushing: effect on bleeding, gingivitis, and supragingival plaque. J Clin Dent 2005;16: 71-7.

33. Bohannan H. Caries distribution and the case for sealants. J Public Health Dent 1983;33:200-4.

34. Ahovuo-Saloranta A, Hiiri A, Nordblad A, Mäkelä M, Worthington HV. Pit and fissure sealants for preventing dental decay in the permanent teeth of children and adolescents. Cochrane Database Syst Rev 2008;(4):CD001830.

35. Knapik JJ, Marshall SW, Lee RB, et al. Mouthguards in sport activities: history, physical properties and injury prevention effectiveness. Sports Med 2007:37; 117-44. 